\title{
"A Study of Vitamin - D Levels in Metabolic Syndrome And its Association with Complications"
}

\author{
Dr. Suganthi.G ${ }^{1}$, Dr. Ganesamoorthy.K, M.D ${ }^{2}$, Dr. Surendran.R ${ }^{3}$ \\ ${ }^{I}$ (Post Graduate, Department Of Medicine, Coimbatore Medical College/ Tamil Nadu Dr.MGR University, \\ India) \\ ${ }^{2}$ (Senior Assistant Professor, Department Of Medicine, Coimbatore Medical College/ Tamil Nadu Dr.MGR \\ University, India) \\ ${ }_{3}^{3}$ (Post Graduate, Department Of Medicine, Coimbatore Medical College/ Tamil Nadu Dr.MGR University, \\ India)
}

\begin{abstract}
The discovery of pleiotropic functions of Vitamin D has led to several studies associating the sunshine vitamin and several diseases. It is the anti inflammatory and immunomodulatory role of the vitamin that links it with metabolic syndrome. It also has anti-proliferative, anti-angiogenesis, anti-thrombotic functions. In our study we are interested in studying the association between Vitamin D levels and components of metabolic syndrome and its complications like stroke and myocardial infarction. It was interesting to find significant association between low levels of vitamin $D$ and components of metabolic syndrome like hypertension, waist circumference, duration of diabetes, triglyceride levels and total cholesterol levels. There was also inverse relationship between vitamin $D$ and vascular complications of metabolic syndrome like myocardial infarction and stroke. We also found that Vitamin D was not significantly associated with fasting blood glucose levels, HDL and LDL cholesterol levels. We find it would be justified to analyse vitamin D insufficiency in patients with metabolic syndrome and supplementation with this vitamin might be of beneficial value in preventing some of the complications of the disease. But the protective and preventive role of Vitamin D in this disease needs further trials to prove its efficiency.
\end{abstract}

Keywords: Vitamin D, Metabolic Syndrome.

\section{Introduction}

Metabolic syndrome consists of a constellation of cardio metabolic disease risk factors. It has been recognized as a global epidemic. Metabolic syndrome is characterised by hyperglycemia especially insulin resistance, central abdominal obesity, dyslipidemia and hypertension. The clinical importance of metabolic syndrome is that it identifies the people at increased long term risk of cardiovascular disease and diabetes mellitus type II. Vitamin D insufficiency has become a common health problem, often unrecognized and untreated. Recently vitamin D, the sunshine vitamin have been reported to be associated with various non skeletal disorders including diabete mellitus, cardiovascular disease and systemic hypertension. Recent research have also linked vitamin D with auto immune diseases, depression and certain types of cancers. The studies have also emphasized on hypovitaminosis D with metabolic syndrome. Metabolic syndrome is a state of low grade systemic inflammation. The immunomodulatory effects and anti inflammatory action of vitamin D links its role with metabolic syndrome. This study aims to analyse the 25 -hydroxy vitamin D status in metabolic syndrome patients and also to correlate its level with individual components and complications of metabolic syndrome.

\subsection{Source of data:}

\section{Methodology}

Patients diagnosed with metabolic syndrome admitted at Coimbatore medical college hospital over a period of 1 year between August 2014 - July 2015.

\subsection{Study design:}

Cross sectional study

\subsection{Sample size:}

100 patients with metabolic syndrome were selected for the study

\subsection{Study population:}

100 patients with metabolic syndrome patients admitted to Coimbatore medical college hospital during the study period were randomly selected as per inclusion and exclusion criteria. Out of these 62 were men and 38 were women. Written informed consent was obtained from each patient. 


\subsection{Inclusion criteria:}

2.5.1. Patients with metabolic syndrome defined using the national cholesterol education progamme, adult treatment panel III (NCEP : ATP III 2001) With three or more of following components

- Central obesity : Waist circumference $>102 \mathrm{~cm}$ (male), $>88 \mathrm{~cm}$ (female)

- Hypertriglyceridemia: Triglycerides $>150 \mathrm{mg} / \mathrm{dl}$ or on specific medication

- Low HDL cholesterol: $<40 \mathrm{mg} / \mathrm{dl}$ for men and $<50 \mathrm{mg} / \mathrm{dl}$ for women or specific medication

- Hypertension: Blood pressure $>130 \mathrm{mmhg}$ systolic or $>85 \mathrm{mmhg}$ diastolic or specific medication.

- Fasting plasma glucose $>100 \mathrm{mg} / \mathrm{dl}$ or specific medication or previously diagnosed type II diabetes mellitus

\subsubsection{Age above 18 years}

2.6. Exclusion criteria:

- Patients with chronic renal failure

- Patients with liver failure

- Patients on treatment with vitamin D

- Malabsorption

- Malignancy

- Infections

\subsection{Data collection:}

The 100 metabolic syndrome patients were included in the study. Detailed history regarding duration of diabetes, hypertension and complications were recorded. A thorough physical examination such as anthropometry, vitals and systemic examination was conducted.The height and weight were recorded and BMI was calculated based on the formula.

$$
\mathrm{BMI}=\frac{\text { Weight in } \mathrm{kg}}{\text { Height in } \mathrm{m}^{2}}
$$

BMI in the range of $<18.5 \mathrm{~kg} / \mathrm{m}^{2}$ was considered as underweight, $18.5-24.9$ was considered as normal, 25-29.9 as overweight, $>30$ as obese. The waist circumference was measured using a standard measuring tape in $\mathrm{cm}$. waist circumference of $>102 \mathrm{~cm}$ in males and $>88 \mathrm{~cm}$ in females was considered as abnormal. Investigations such as fasting blood sugar, lipid profile (Triglycerides, HDL, Total cholesterol) were done and finding were recovered.

Serum vitamin D levels was measured and interpreted as follows $<20 \mathrm{ng} / \mathrm{ml}-$ deficient, $21-29 \mathrm{ng} / \mathrm{ml}-$ insufficient and $30 \mathrm{ng} / \mathrm{ml}$ - sufficient

\subsection{Stastical method}

Stastical method analysis was done using chi square test and ANOVA test. Stastical results were considered significant at $\mathrm{p}<0.05$

III. Results

Table No : 1 - Age Distribution

\begin{tabular}{|c|c|c|}
\hline & & $3 \%$ \\
\hline $21-30 \mathrm{yrs}$ & 3 & $13 \%$ \\
\hline $31-40 \mathrm{yrs}$ & 13 & $28 \%$ \\
\hline $41-50 \mathrm{yrs}$ & 28 & $31 \%$ \\
\hline $51-60 \mathrm{yrs}$ & 31 & $18 \%$ \\
\hline $61-70 \mathrm{yrs}$ & 18 & $7 \%$ \\
\hline$>70 \mathrm{yrs}$ & 7 & $\mathbf{1 0 0 \%}$ \\
\hline Total & $\mathbf{1 0 0}$ & \\
\hline
\end{tabular}

Chart No : 1 - Age Distribution

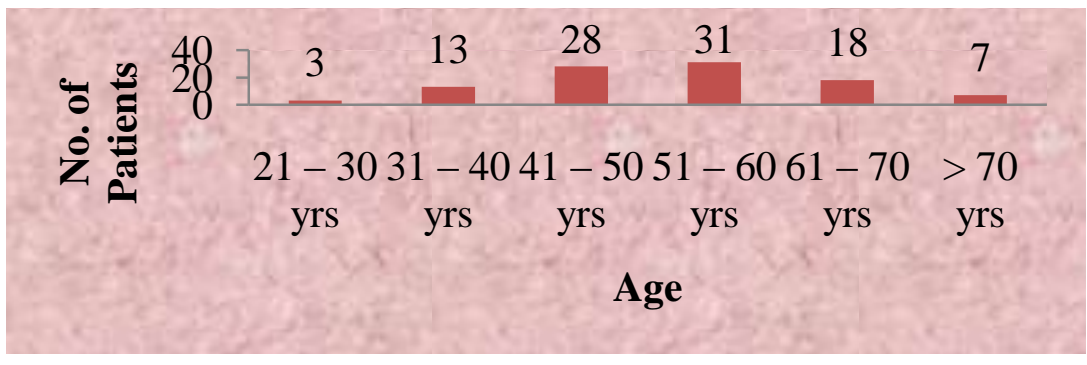


Patients age ranged from 21-80 years. Maximum number of cases were in the age group of 51-60 years $(31 \%)$ and $28 \%$ of cases were in the age group of $41-50$ years.

Inference : Majority of patients were in the $5^{\text {th }}$ and $6^{\text {th }}$ decade.

Table No : 2 - Sex

\begin{tabular}{|c|c|c|}
\hline Sex & No. of Patients & $\%$ \\
\hline Male & 62 & $62 \%$ \\
\hline Female & 38 & $38 \%$ \\
\hline Total & 100 & $100 \%$ \\
\hline
\end{tabular}

Chart No : 2 - Sex

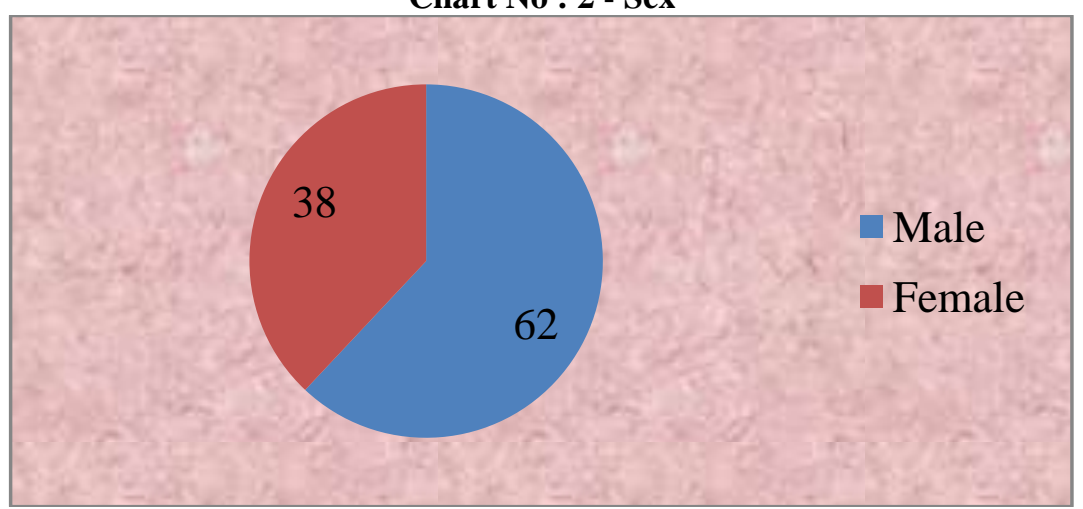

Out of 100 patients, $62(62 \%)$ were males and 38 patients $(38 \%)$ were females

Table No : 3 - Components Of Metabolic Syndrome

\begin{tabular}{|l|c|c|}
\hline & & \\
\hline Waist Circumference & 77 & $77 \%$ \\
\hline Hypertension & 82 & $82 \%$ \\
\hline Triglycerides $>150 \mathrm{mg} / \mathrm{dl}$ & 74 & $74 \%$ \\
\hline Fasting blood sugar & 83 & $73 \%$ \\
\hline HDL & 74 & $74 \%$ \\
\hline Total & $\mathbf{1 0 0}$ & $\mathbf{1 0 0 \%}$ \\
\hline
\end{tabular}

Chart No : 3 - Components Of Metabolic Syndrome

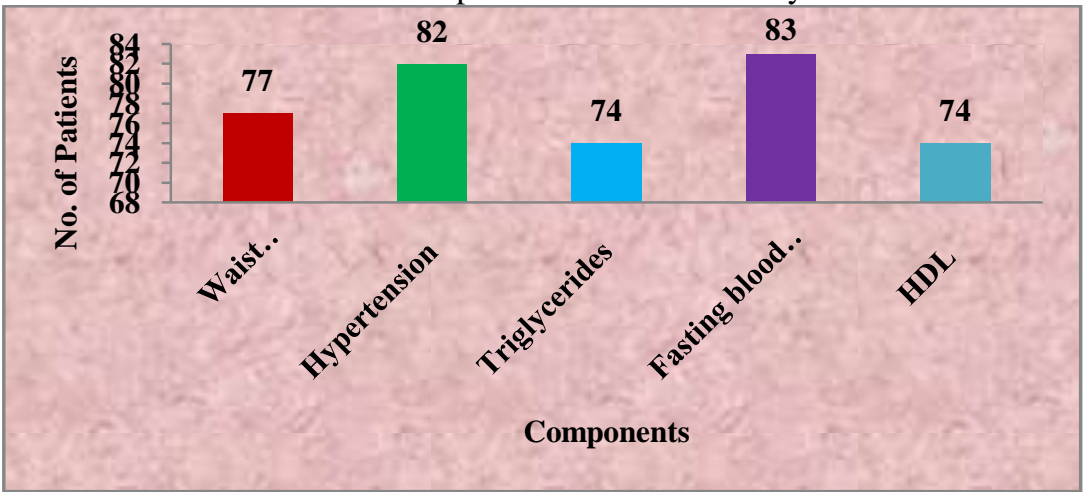

The commonest component of metabolic syndrome was observed to be fasting blood sugar abnormality $(83 \%)$ followed by hypertension (82\%). Waist circumference $(77 \%)$, low HDL (74\%), triglycerides (74\%.)

Table No : 4 - Fasting Blood Sugar

\begin{tabular}{|c|c|c|}
\hline & & \\
\hline Presented with DM & 60 & $60 \%$ \\
\hline New & 23 & $23 \%$ \\
\hline Absent & 17 & $17 \%$ \\
\hline Total & 100 & $100 \%$ \\
\hline
\end{tabular}


Chart No : 4 - Fasting Blood Sugar

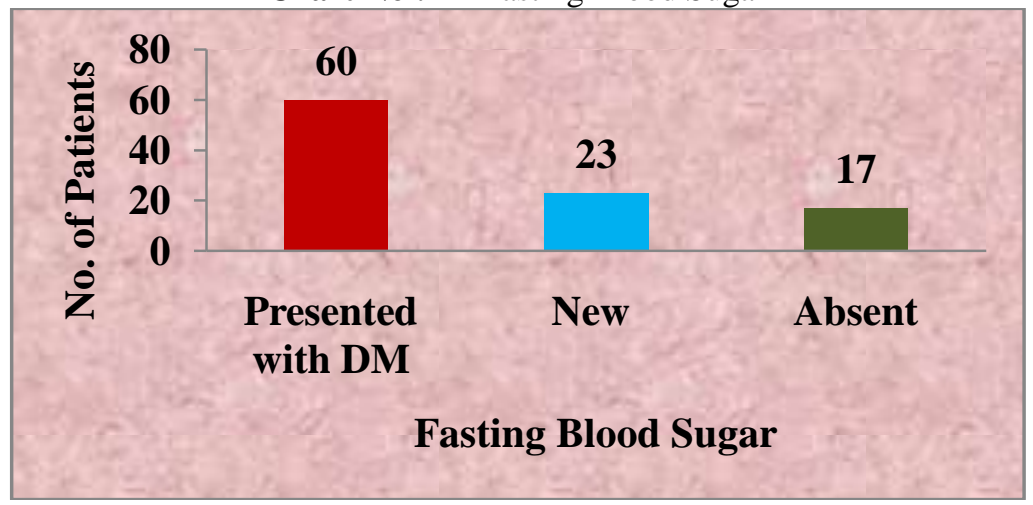

$60 \%$ of the patients were known diabetic, $23 \%$ of patients had fasting blood sugar abnormality and $17 \%$ had normal fasting blood sugar levels.

Table No : 5 - Blood Pressure

\begin{tabular}{|c|c|c|}
\hline & & \\
\hline Presented with SHT & 68 & $68 \%$ \\
\hline Newly detected & 14 & $14 \%$ \\
\hline Absent & 18 & $18 \%$ \\
\hline Total & 100 & $100 \%$ \\
\hline
\end{tabular}

Chart No : 5 - Blood Pressure

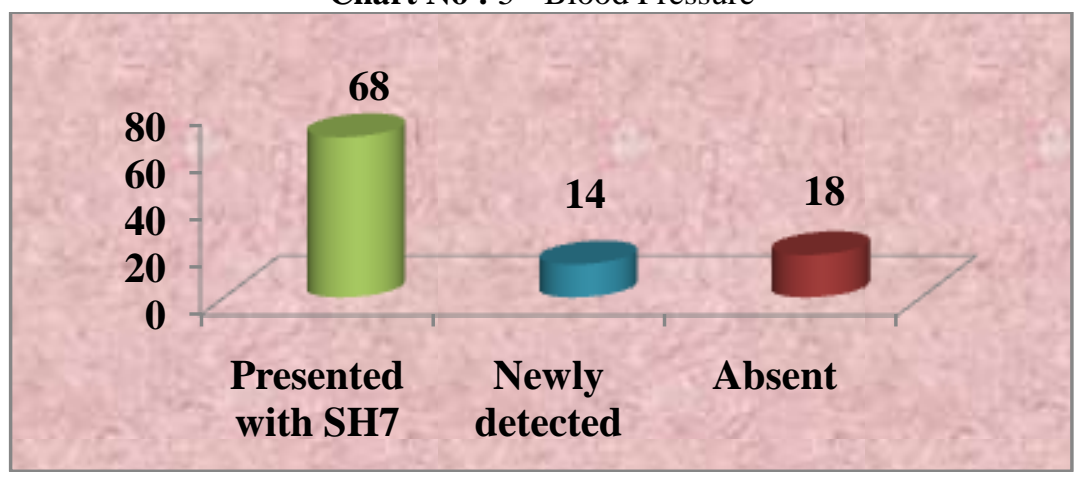

$68 \%$ of the patients were known hypertensive, $14 \%$ were newly detected and $18 \%$ of patients had normal blood pressure levels.

Table No : 6 - High Density Lipoprotein

\begin{tabular}{|c|c|c|}
\hline & & \\
\hline Normal & 26 & $26 \%$ \\
\hline Abnormal & 74 & $74 \%$ \\
\hline Total & 100 & $100 \%$ \\
\hline
\end{tabular}

Chart No : 6 - High Density Lipoprotein

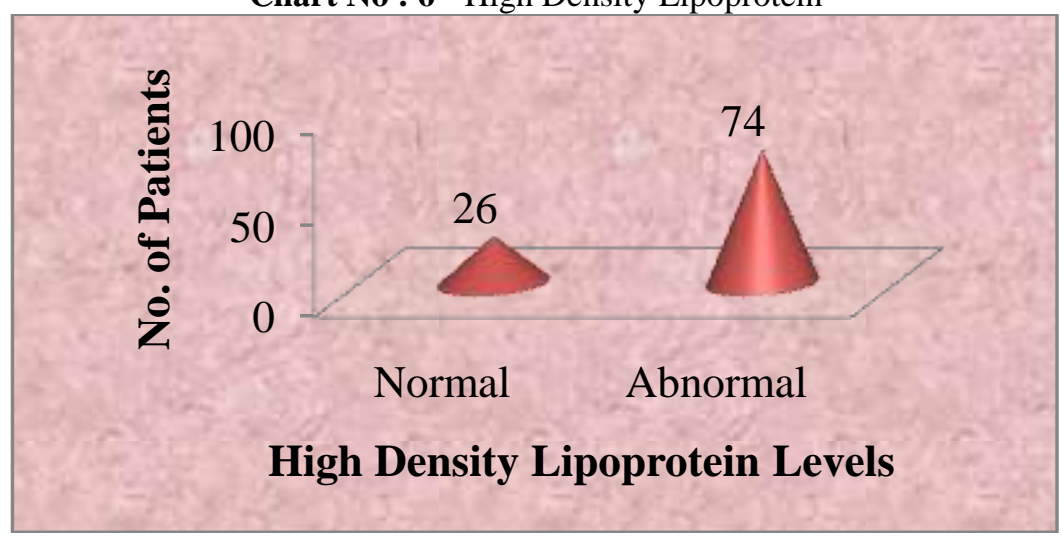


$74 \%$ of patients had abnormal High Density Lipoprotein cholesterol levels and $26 \%$ of patients had normal High Density Lipoprotein levels.

Table No : 7 - Low Density Lipoprotein

\begin{tabular}{|c|c|c|}
\hline & & \\
\hline$<100$ & 7 & $7 \%$ \\
\hline$>100$ & 93 & $93 \%$ \\
\hline Total & 100 & $100 \%$ \\
\hline
\end{tabular}

Chart No : 7 - Low Density Lipoprotein

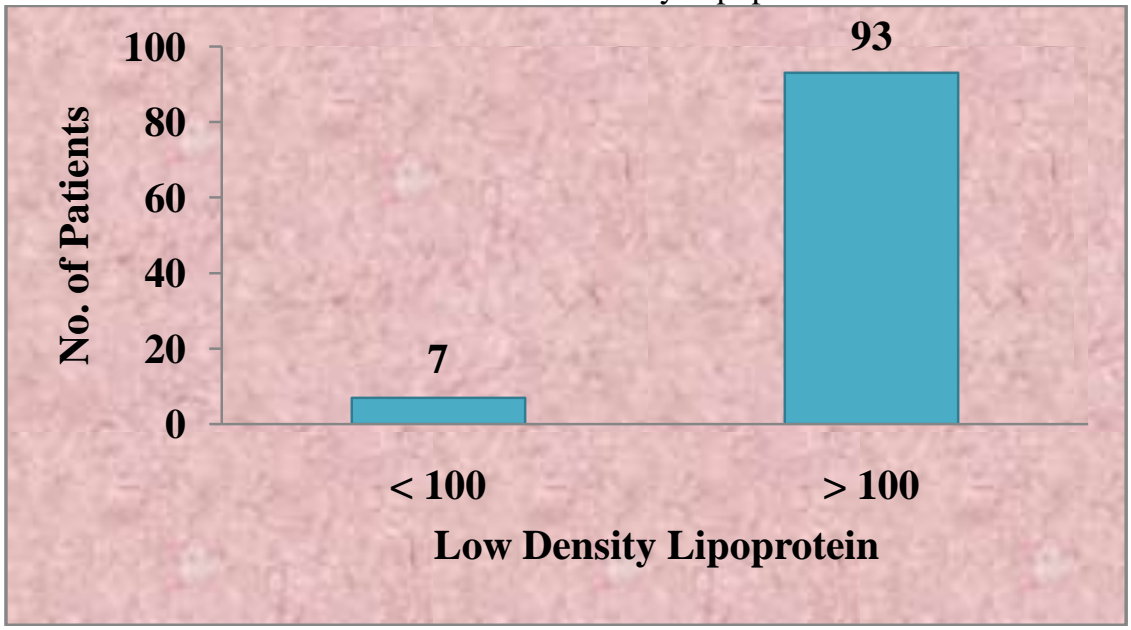

93\% of patients had abnormal Low Density Lipoprotein levels> $100 \mathrm{mgldl}$ and $7 \%$ of patients had normal Low Density Lipoprotein levels.

Table No : 8 - Serum Cholesterol

\begin{tabular}{|c|c|c|}
\hline & & \\
\hline$<200$ & 22 & $22 \%$ \\
\hline$>200$ & 78 & $78 \%$ \\
\hline Total & 100 & $100 \%$ \\
\hline
\end{tabular}

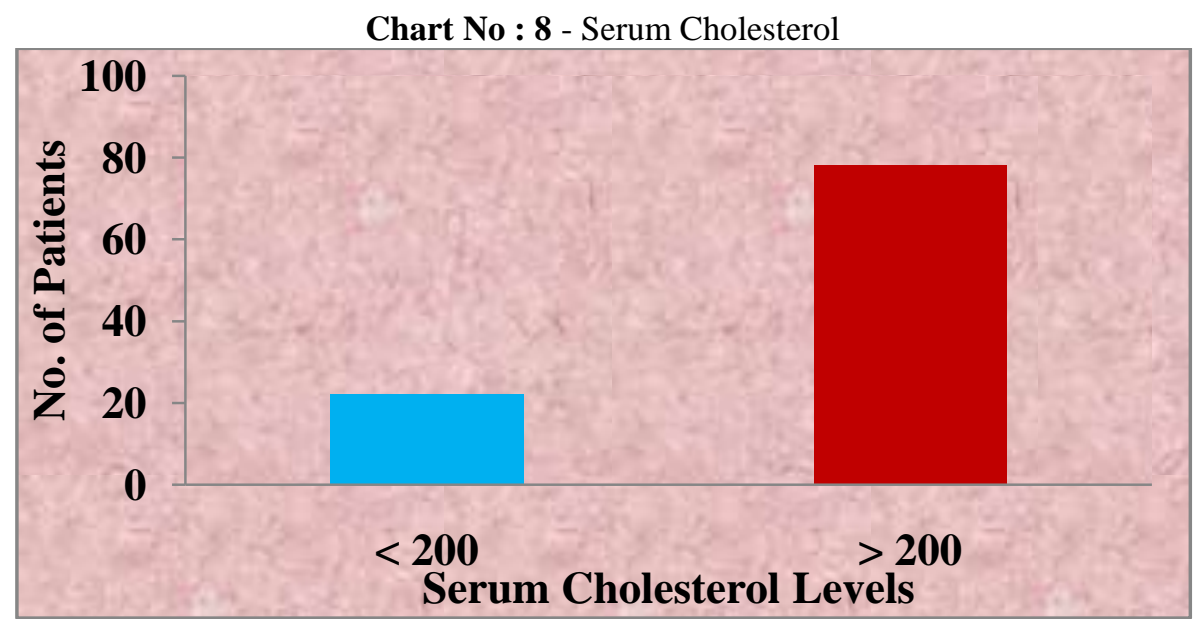

$78 \%$ of patients had total cholesterol more than $200 \mathrm{mg} / \mathrm{dl}$ and $22 \% \mathrm{had}<200 \mathrm{mg} / \mathrm{dl}$.

Table No : 9 - Body Mass Index

\begin{tabular}{|l|c|c|}
\hline \multicolumn{2}{|c|}{ Table No : 9 - Body Mass Index } \\
\hline Normal (18.5-24.9) & 13 & $13 \%$ \\
\hline Overweight (24.9-29.9) & 54 & $54 \%$ \\
\hline Obese (>29.9) & 33 & $33 \%$ \\
\hline Total & $\mathbf{1 0 0}$ & $\mathbf{1 0 0 \%}$ \\
\hline
\end{tabular}


Chart No : 9 - Body Mass Index

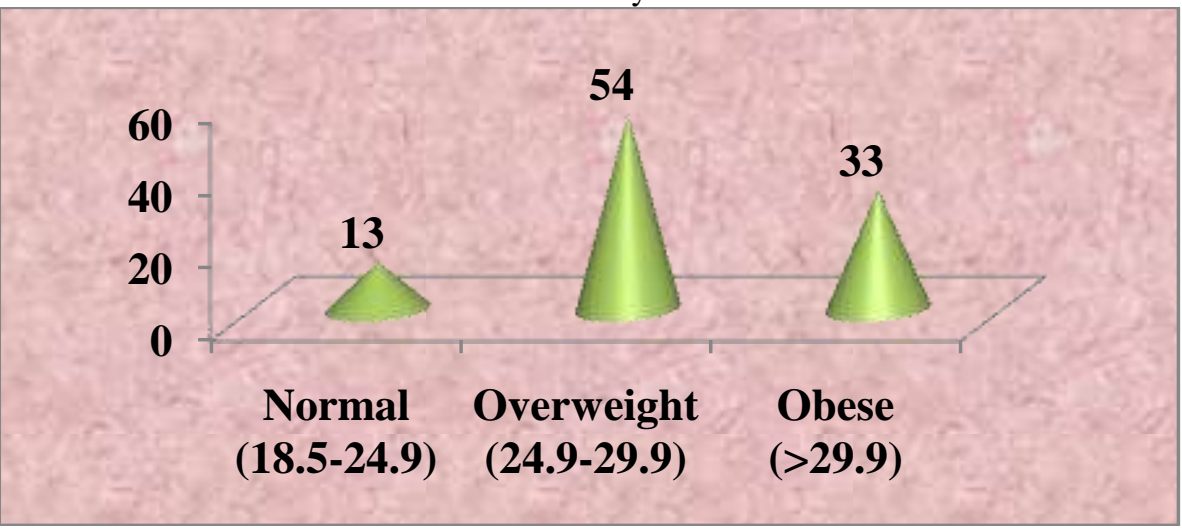

$13 \%$ of patients were of normal weight,54\% of them were overweight and $33 \%$ were obese.

Table No : 10 - Serum Vitamin-D

\begin{tabular}{|l|c|c|}
\hline & & \\
\hline Deficiency (<20) & 82 & $82.0 \%$ \\
\hline Insufficiency (21-29) & 12 & $12.0 \%$ \\
\hline Normal $(>30)$ & 6 & $6.0 \%$ \\
\hline Total & $\mathbf{1 0 0}$ & $\mathbf{1 0 0 \%}$ \\
\hline
\end{tabular}

Chart No : 10 - Serum Vitamin-D

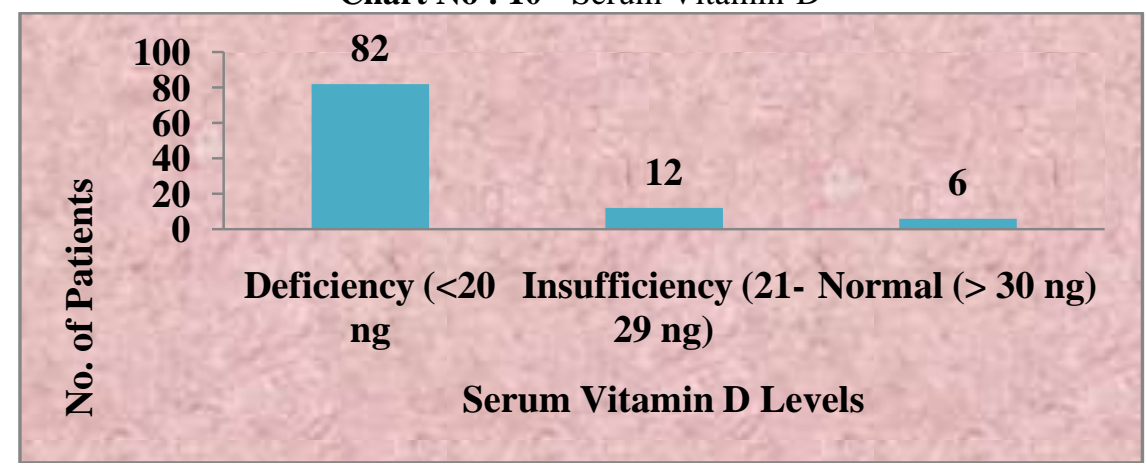

$82 \%$ of patients had deficient $(<20 \mathrm{ng} / \mathrm{dl})$ vitamin D levels, $12 \%$ of patients had insufficient levels (21$29 \mathrm{ng} / \mathrm{dl}$ ) and $6 \%$ of patients had sufficient (> $30 \mathrm{ng} / \mathrm{dl}$ ) levels of vitamin D.

Table no.11 Number of components of metabolic syndrome

\begin{tabular}{|l|c|c|}
\hline & & \\
\hline 3 & 35 & $35 \%$ \\
\hline 4 & 46 & $46 \%$ \\
\hline 5 & 19 & $19 \%$ \\
\hline Total & $\mathbf{1 0 0}$ & $\mathbf{1 0 0 \%}$ \\
\hline
\end{tabular}

Chartno. 11 number Of Components Of Metabolic Syndrome

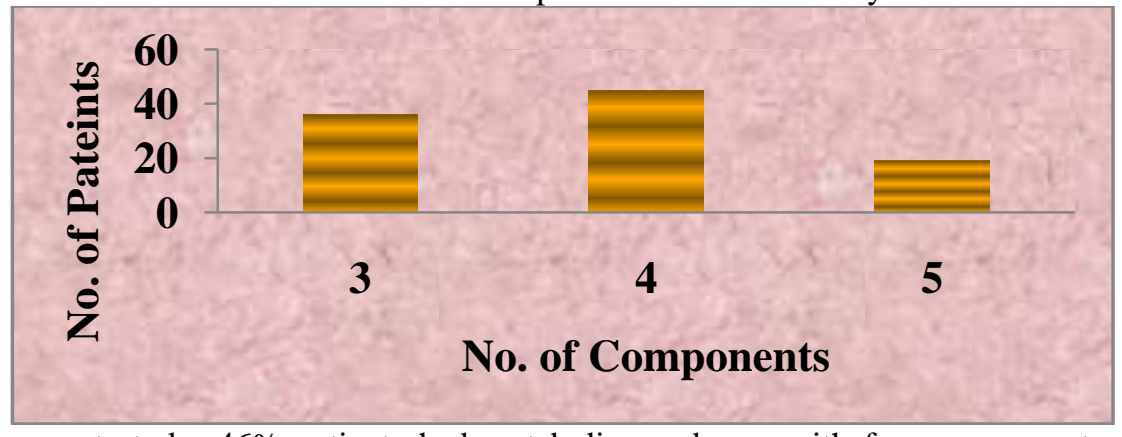

In the present study, $46 \%$ patients had metabolic syndrome with four components and $35 \%$ had 3 components and $19 \%$ had 5 components of metabolic syndrome. 
“A Study Of Vitamin - D Levels In Metabolic Syndrome And Its Association...

Table No.12 Complications Of Metabolic Syndrome

\begin{tabular}{|c|c|c|c|}
\hline Complications & & No. of Patients & $\%$ \\
\hline \multirow{2}{*}{ Stroke } & Yes & 18 & $18 \%$ \\
\cline { 2 - 4 } & No & 82 & $82 \%$ \\
\hline \multirow{2}{*}{ Myocardial Infection } & Yes & 20 & $20 \%$ \\
\cline { 2 - 4 } & No & 80 & $80 \%$ \\
\hline
\end{tabular}

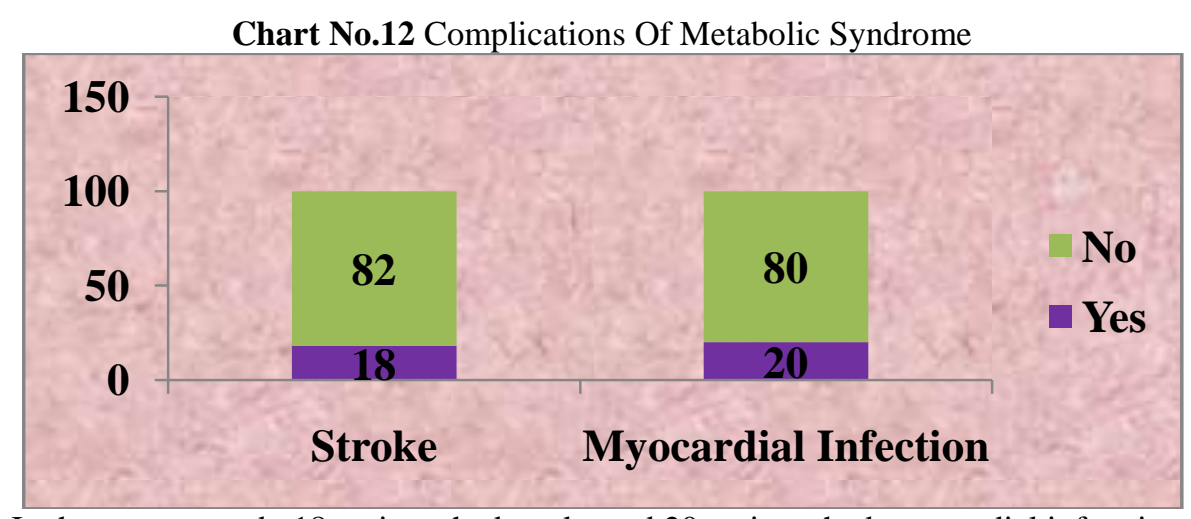

In the present study, 18 patients had stroke and 20 patients had myocardial infarction.

Table:13 Correlation Of Vitamin D Levels With Age

\begin{tabular}{|c|c|c|c|c|}
\hline & \multirow{2}{*}{ Age } & \multicolumn{3}{|c|}{ VITAMIN D } \\
\hline & & Mean & S.D & No. \\
\hline \multirow{6}{*}{ Age } & 21-30 YRS & 22.33 & 6.03 & 3 \\
\hline & $31-40$ YRS & 17.92 & 7.16 & 13 \\
\hline & $41-50$ YRS & 17.18 & 5.81 & 28 \\
\hline & 51-60 YRS & 15.00 & 5.76 & 31 \\
\hline & 61-70 YRS & 12.61 & 5.74 & 18 \\
\hline & $71-80$ YRS & 15.43 & 7.46 & 7 \\
\hline & Total & 15.81 & 6.30 & 100 \\
\hline
\end{tabular}

Anova For Vitamin D

\begin{tabular}{|l|c|c|c|c|c|}
\hline & Sum of Squares & df & Mean Square & F & Sig. \\
\hline Between Groups & 443.701 & 5 & 88.740 & 2.393 & $<0.05$ \\
\hline Within Groups & $3,485.689$ & 94 & 37.082 & & \\
\hline Total & $\mathbf{3 , 9 2 9 . 3 9 0}$ & $\mathbf{9 9}$ & & & \\
\hline
\end{tabular}

We observed vitamin D levels were less in age group of $5^{\text {th }}, 6^{\text {th }} \& 7^{\text {th }}$ decade.ANOVA test shows that there is significant difference in the mean vitamin D levels with age groups. There is significant decrease in vitamin D levels with increasing age.

Table : 14 - Correlation Of Vitamin D With Gender

\begin{tabular}{|l|l|r|r|r|}
\hline \multicolumn{2}{|c|}{} & \multicolumn{3}{|c|}{ VITAMIN D } \\
\cline { 3 - 5 } \multicolumn{2}{c|}{} & Mean & \multicolumn{1}{|c|}{ S.D } & No. \\
\hline Gender & Male & 16.48 & 6.65 & 62 \\
\cline { 2 - 5 } & Female & 14.71 & 5.59 & 38 \\
\hline Total & 15.81 & 6.30 & 100 \\
\hline
\end{tabular}

t-test for Equality of Means

\begin{tabular}{|c|c|c|}
\hline $\mathrm{t}$ & $\mathrm{df}$ & Sig. \\
\hline 1.372 & 98 & Ns \\
\hline
\end{tabular}

The mean vitamin D levels among men was $16.48 \mathrm{ng} / \mathrm{ml}$ and among women was 14.71.we observed that there is no significant difference in vitamin D levels among men and women in our study.

Table : 15 - Bmi

\begin{tabular}{|c|c|c|c|c|c|c|c|c|c|}
\hline & \multirow{3}{*}{ BMI } & \multicolumn{6}{|c|}{ VITAMIN D } & \multicolumn{2}{|c|}{ TOTAL } \\
\hline & & \multicolumn{2}{|c|}{$\begin{array}{c}\text { Deficient } \\
(<=20)\end{array}$} & \multicolumn{2}{|c|}{$\begin{array}{c}\text { Insufficient } \\
(21-29)\end{array}$} & \multicolumn{2}{|c|}{$\begin{array}{c}\text { Sufficient } \\
(>=\mathbf{3 0})\end{array}$} & \multirow[b]{2}{*}{ No. } & \multirow[b]{2}{*}{$\%$} \\
\hline & & No. & $\%$ & No. & $\%$ & No. & $\%$ & & \\
\hline \multirow{3}{*}{ BMI } & Normal (18.5-24.9) & 7 & 53.8 & 4 & 30.8 & 2 & 15.4 & 13 & 100.0 \\
\hline & Overweight (24.9-29.9) & 45 & 83.3 & 7 & 13.0 & 2 & 3.7 & 54 & 100.0 \\
\hline & Obese $(>29.9)$ & 30 & 90.9 & 1 & 3.0 & 2 & 6.1 & 33 & 100.0 \\
\hline & TOTAL & 82 & 82.0 & 12 & 12.0 & 6 & 6.0 & 100 & 100.0 \\
\hline
\end{tabular}




\begin{tabular}{|l|c|c|c|c|} 
Chi-Square Test \\
\hline Chi-Square & Value & df & Sig. & P value \\
\hline & 10.041 & 4 & .040 & $\mathrm{P}<0.05$ \\
\hline
\end{tabular}

Table no : 16

Correlation of vitamin d levels with duration of diabetes mellitus

\begin{tabular}{|c|c|c|c|c|}
\hline \multicolumn{2}{|c|}{} & \multicolumn{3}{|c|}{ VITAMIN D } \\
\cline { 2 - 5 } Diabetes mellitus-Duration & $1-5$ YRS & 17.29 & S.D & No. \\
\hline & $6-10$ YRS & 14.00 & 6.78 & 21 \\
\cline { 2 - 5 } & $>10$ YRS & 12.42 & 5.35 & 12 \\
\hline Total & $\mathbf{1 4 . 8 3}$ & 3.80 & $\mathbf{5 0}$ \\
\hline
\end{tabular}

Anova For Vitamin D

\begin{tabular}{|c|c|c|c|c|c|}
\hline & Sum of Squares & df & Mean Square & F & Sig. \\
\hline Between Groups & 215.131 & 2 & 107.565 & 3.367 & $<0.05$ \\
\hline Within Groups & $1,821.202$ & 57 & 31.951 & & \\
\hline Total & $\mathbf{2 , 0 3 6 . 3 3 3}$ & $\mathbf{5 9}$ & & & \\
\hline
\end{tabular}

ANOVA test shows that there is significant decrease in vitamin D levels with increase in duration of diabetes mellitus. $(\mathrm{p}<0.05)$

Table No : 17 Correlation Of Vitamin D With Duration Of Hypertension

\begin{tabular}{|c|c|c|c|c|}
\hline & & \multicolumn{3}{|c|}{ VITAMIN D } \\
\hline & & Mean & S.D & No. \\
\hline \multirow{3}{*}{$\begin{array}{c}\text { SYSTEMIC HYPERTENSION- } \\
\text { Duration }\end{array}$} & $1-5$ YRS & 16.13 & 6.11 & 40 \\
\hline & 6-10 YRS & 13.76 & 5.47 & 21 \\
\hline & $>10$ YRS & 14.00 & 6.81 & 7 \\
\hline \multicolumn{2}{|l|}{ Total } & 15.18 & 6.01 & 68 \\
\hline
\end{tabular}

Anova For Vitamin D

\begin{tabular}{|l|r|r|r|r|c|}
\hline & Sum of Squares & df & Mean Square & F & Sig. \\
\hline Between Groups & 87.698 & 2 & 43.849 & 1.222 & Ns \\
\hline Within Groups & $2,332.185$ & 65 & 35.880 & & \\
\hline Total & $\mathbf{2 , 4 1 9 . 8 8 2}$ & $\mathbf{6 7}$ & & & \\
\hline
\end{tabular}
hypertension.

ANOVA test shows there is no significant association between vitamin D levels and duration of

Table no : $\mathbf{1 8}$ correlation of vitamin d levels with waist circumference:

\begin{tabular}{|c|c|c|c|c|c|c|c|c|c|}
\hline & & \multicolumn{6}{|c|}{ VITAMIN D } & \multicolumn{2}{|c|}{ TOTAL } \\
\hline & & \multicolumn{2}{|c|}{$\begin{array}{l}\text { Deficient } \\
(<=20)\end{array}$} & \multicolumn{2}{|c|}{\begin{tabular}{|c|} 
Insufficient \\
$(21-29)$
\end{tabular}} & \multicolumn{2}{|c|}{$\begin{array}{c}\text { Sufficient } \\
(>=30)\end{array}$} & \multirow[t]{2}{*}{ No. } & \multirow[t]{2}{*}{$\%$} \\
\hline & & No. & $\%$ & No. & $\%$ & No. & $\%$ & & \\
\hline \multirow{2}{*}{ WC } & Abnormal & 65 & 84.4 & 10 & 13.0 & 2 & 2.6 & 77 & 100.0 \\
\hline & Normal & 17 & 73.9 & 2 & 8.7 & 4 & 17.4 & 23 & 100.0 \\
\hline \multicolumn{2}{|c|}{ TOTAL } & 82 & 82.0 & 12 & 12.0 & 6 & 6.0 & 100 & 100.0 \\
\hline
\end{tabular}

Chi-Square Test

\begin{tabular}{|c|c|c|c|}
\hline & Value & df & Sig. \\
\hline Chi-Square & 6.970 & 2 & $<0.05$ \\
\hline
\end{tabular}

When levels of vitamin D were compared with waist circumference, most of the patients with abnormal waist circumference had deficient levels(84.4\%),10 patients(13\%) had insufficient levels and only 2 patients $(2.6 \%)$ had sufficient levels.(p<0.05, significant)

Table No : 19 Correlation Of Vitamin D Levels With Fasting Blood Sugar

\begin{tabular}{|c|c|c|c|c|c|c|c|c|c|}
\hline & & \multicolumn{6}{|c|}{ VITAMIN D } & \multicolumn{2}{|c|}{ TOTAL } \\
\hline & & \multicolumn{2}{|c|}{$\begin{array}{c}\text { Deficient } \\
(<=20)\end{array}$} & \multicolumn{2}{|c|}{$\begin{array}{c}\text { Insufficient } \\
(21-29)\end{array}$} & \multicolumn{2}{|c|}{$\begin{array}{c}\text { Sufficient } \\
(>=30)\end{array}$} & \multirow[t]{2}{*}{ No. } & \multirow[t]{2}{*}{$\%$} \\
\hline & & No. & $\%$ & No. & $\%$ & No. & $\%$ & & \\
\hline FBS & $\begin{array}{c}\text { Abnormal } \\
(>100)\end{array}$ & 71 & 85.5 & 6 & 8.4 & 5 & 6.0 & 83 & 100.0 \\
\hline
\end{tabular}


“A Study Of Vitamin - D Levels In Metabolic Syndrome And Its Association...

\begin{tabular}{|c|c|c|c|c|c|c|c|c|c|}
\hline $\begin{array}{c}\text { Normal } \\
(<=100)\end{array}$ & 11 & 64.7 & 6 & 29.4 & 1 & 5.9 & 17 & 100.0 \\
\hline TOTAL & $\mathbf{8 2}$ & $\mathbf{8 2 . 0}$ & $\mathbf{1 2}$ & $\mathbf{1 2 . 0}$ & $\mathbf{6}$ & $\mathbf{6 . 0}$ & $\mathbf{1 0 0}$ & $\mathbf{1 0 0 . 0}$ \\
\hline
\end{tabular}

Chi-Square Test

\begin{tabular}{|c|c|c|c|}
\hline & Value & df & Sig. \\
\hline Chi-Square & 5.922 & 2 & Ns \\
\hline
\end{tabular}

When levels of vitamin D were compared with, Fasting blood sugar,most of the patients with fasting sugar abnormality had deficient levels $(85.5 \%), 6$ patients $(8.4 \%)$ had insufficient levels and only 5 patients $(6 \%)$ had sufficient levels.(p>0.05,insignificant)

Table no : 20 correlation of vitamin d levels with elevated blood pressure:

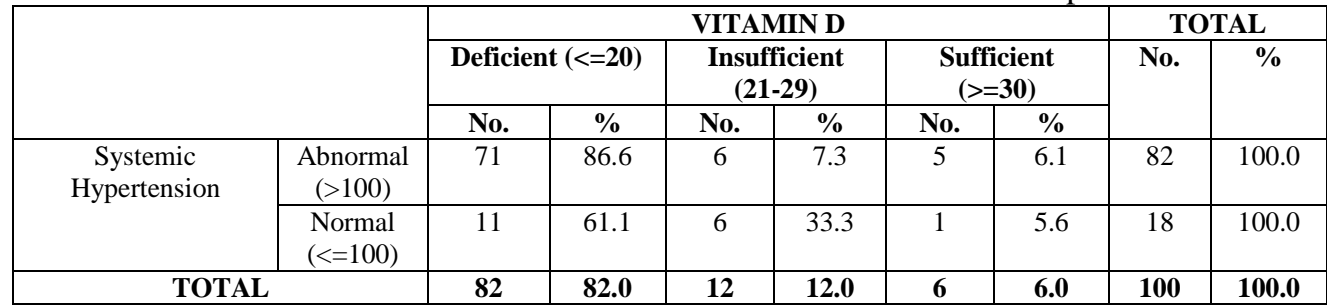

Chi-Square Test

\begin{tabular}{|l|c|c|c|}
\hline & Value & df & Sig. \\
\hline Chi-Square & 9.501 & 2 & $<0.01$ \\
\hline
\end{tabular}

When levels of vitamin D were compared with, blood pressure, most of the patients with elevated blood pressure had deficient levels(86.6\%),6 patients(7.3\%) had insufficient levels and only 5 patients $(6.1 \%)$ had sufficient levels. $(\mathrm{p}<0.01$, significant)

Table no : 21 correlation of vitamin d levels with triglycerides:

\begin{tabular}{|c|c|c|c|c|c|c|c|c|c|}
\hline & \multicolumn{6}{|c|}{ VITAMIN D } & \multicolumn{2}{|c|}{ TOTAL } \\
\hline & & \multicolumn{2}{|c|}{$\begin{array}{c}\text { Deficient } \\
(<=20)\end{array}$} & \multicolumn{2}{|c|}{$\begin{array}{c}\text { Insufficient } \\
(21-29)\end{array}$} & \multicolumn{2}{|c|}{$\begin{array}{c}\text { Sufficient } \\
(>=30)\end{array}$} & \multirow[t]{2}{*}{ No. } & \multirow[t]{2}{*}{$\%$} \\
\hline & & No. & $\%$ & No. & $\%$ & No. & $\%$ & & \\
\hline \multirow[t]{2}{*}{ TGL } & $\begin{array}{c}\text { Abnormal } \\
(>=150)\end{array}$ & 65 & 87.8 & 5 & 6.8 & 4 & 5.4 & 74 & 100.0 \\
\hline & Normal $(<150)$ & 17 & 65.4 & 7 & 26.9 & 2 & 7.7 & 26 & 100.0 \\
\hline & TOTAL & 82 & 82.0 & 12 & 12.0 & 6 & 6.0 & 100 & 100.0 \\
\hline
\end{tabular}

Chi-Square Tests

\begin{tabular}{|l|c|c|c|c|}
\hline & Value & df & Sig. & P value \\
\hline Pearson Chi-Square & $7.871^{\text {a }}$ & 2 & .020 & $\mathrm{P}<0.05$ \\
\hline
\end{tabular}

Out of 82 patients with deficient vitamin D Levels, 65 patients $(87.8 \%)$ had $>150 \mathrm{mg} / \mathrm{dl}, 17$ patients $(65.4 \%)$ had $<150 \mathrm{mg} / \mathrm{dl}$. Among 12 patients with insufficient levels,5(6.8\%) had abnormal triglycerides and7 $(26.9 \%)$ had normal triglyceride levels. Out of 6 patients with sufficient levels, 4 patients $(5.4 \%)$ had triglycerides $>150 \mathrm{mg} / \mathrm{dl}$ and $2(7.7 \%)$ had levels $<150 \mathrm{mg} / \mathrm{dl} .(\mathrm{p}<0.05)$.

Table No : 22 Correlation Of Vitamin D Levels With Hdl

\begin{tabular}{|c|c|c|c|c|c|c|c|c|}
\hline & \multicolumn{6}{|c|}{ VITAMIN D } & \multicolumn{2}{|c|}{ TOTAL } \\
\hline & \multicolumn{2}{|c|}{$\begin{array}{l}\text { Deficient } \\
(<=20)\end{array}$} & \multicolumn{2}{|c|}{\begin{tabular}{|c|} 
Insufficient \\
$(21-29)$
\end{tabular}} & \multicolumn{2}{|c|}{$\begin{array}{c}\text { Sufficient } \\
(>=30)\end{array}$} & \multirow[b]{2}{*}{ No. } & \multirow[b]{2}{*}{$\%$} \\
\hline & No. & $\%$ & No. & $\%$ & No. & $\%$ & & \\
\hline \begin{tabular}{l|l|} 
& Abnormal \\
\end{tabular} & 59 & 79.7 & 10 & 13.5 & 5 & 6.8 & 74 & 100.0 \\
\hline HDL & 23 & 88.5 & 2 & 7.7 & 1 & 3.8 & 26 & 100.0 \\
\hline TOTAL & 82 & 82.0 & 12 & 12.0 & 6 & 6.0 & 100 & 100.0 \\
\hline
\end{tabular}

\begin{tabular}{|l|c|c|c|c|}
\hline \multicolumn{1}{|c|}{ Chi-Square Test } \\
\hline Chi-Square & Value & df & Sig. & P value \\
\hline
\end{tabular}

Out of 82 patients with deficient vitamin D Levels,59 patients (79.7\%) had abnormal HDL values,23 patients $(88.5 \%)$ had normal levels. Among 12 patients with insufficient levels,10(13.5\%) had abnormal HDL 
and2 $(7.7 \%)$ had normal HDL level. Out of 6 patients with sufficient levels, 5 patients(6.8\%) had abnormal HDL and $1(3.8 \%)$ had normal HDL. $(\mathrm{p}>0.05)$.

Table No : 23 Correlation Of Vitamin D Levels With Ldl

\begin{tabular}{|c|c|c|c|c|c|c|c|c|c|}
\hline & \multicolumn{6}{|c|}{ VITAMIN D } & \multicolumn{2}{|c|}{ TOTAL } \\
\hline & & \multicolumn{2}{|c|}{$\begin{array}{c}\text { Deficient } \\
(<=20)\end{array}$} & \multicolumn{2}{|c|}{$\begin{array}{c}\text { Insufficient } \\
(21-29)\end{array}$} & \multicolumn{2}{|c|}{$\begin{array}{c}\text { Sufficient } \\
(>=30)\end{array}$} & \multirow[t]{2}{*}{ No. } & \multirow[t]{2}{*}{$\%$} \\
\hline & & No. & $\%$ & No. & $\%$ & No. & $\%$ & & \\
\hline \multirow{2}{*}{ LDL } & $\begin{array}{c}\text { Abnormal } \\
(>=100)\end{array}$ & 76 & 81.7 & 11 & 11.8 & 6 & 6.5 & 93 & 100.0 \\
\hline & $\begin{array}{c}\text { Normal } \\
(<100)\end{array}$ & 6 & 85.7 & 1 & 14.3 & & & 7 & 100.0 \\
\hline \multicolumn{2}{|c|}{ TOTAL } & 82 & 82.0 & 12 & 12.0 & 6 & 6.0 & 100 & 100.0 \\
\hline
\end{tabular}

chi-square test

\begin{tabular}{|l|c|c|c|}
\hline & Value & df & Sig. \\
\hline Chi-Square & .497 & 2 & Ns \\
\hline
\end{tabular}

Out of 82 patients with deficient vitamin D Levels,76 patients $(81.7 \%)$ had abnormal LDL values,6 patients $(85.7 \%)$ had normal levels. Among 12 patients with insufficient levels,11(11.8\%) had abnormal LDL and $1(14.3 \%)$ had normal LDL level. Out of 6 patients with sufficient levels, all 6 had patients $(6.5 \%)$ had abnormal HDL. $(\mathrm{p}>0.05)$.

Table No : 24 Correlation Of Vitamin D Levels With Total Cholesterol

\begin{tabular}{|c|c|c|c|c|c|c|c|c|c|}
\hline & & \multicolumn{6}{|c|}{ VITAMIN D } & \multicolumn{2}{|c|}{ TOTAL } \\
\hline & & \multicolumn{2}{|c|}{$\begin{array}{c}\text { Deficient } \\
(<=20)\end{array}$} & \multicolumn{2}{|c|}{$\begin{array}{c}\text { Insufficient } \\
(21-29)\end{array}$} & \multicolumn{2}{|c|}{$\begin{array}{c}\text { Sufficient } \\
(>=30)\end{array}$} & \multirow[t]{2}{*}{ No. } & \multirow[t]{2}{*}{$\%$} \\
\hline & & No. & $\%$ & No. & $\%$ & No. & $\%$ & & \\
\hline \multirow{2}{*}{$\mathrm{TC}$} & $\begin{array}{c}\text { Abnormal } \\
(>=200)\end{array}$ & 67 & 85.9 & 6 & 7.7 & 5 & 6.4 & 78 & 100.0 \\
\hline & $\begin{array}{c}\text { Normal } \\
(<200)\end{array}$ & 15 & 68.2 & 6 & 27.3 & 1 & 4.5 & 22 & 100.0 \\
\hline \multicolumn{2}{|c|}{ TOTAL } & 82 & 82.0 & 12 & 12.0 & 6 & 6.0 & 100 & 100.0 \\
\hline
\end{tabular}

Chi-Square Test
\begin{tabular}{|l|c|c|c|c|}
\hline & Value & df & Sig. & P value \\
\hline Chi-Square & $6.239^{\mathrm{a}}$ & 2 & $*$ & $\mathrm{P}<0.05$ \\
\hline
\end{tabular}

Out of 82 patients with deficient vitamin D Levels, 67 patients $(85.9 \%)$ had abnormal total cholesterol values, 15 patients $(68.2 \%)$ had normal levels. Among 12 patients with insufficient levels,6 (7.7\%) had abnormal total cholesterol and $6(27.3 \%)$ had normal total cholesterol level. Out of 6 patients with sufficient levels,5 had patients $(6.4 \%)$ had abnormal HDL and 1 (4.5\%)had normal levels. $(\mathrm{p}<0.05)$.

Table No : 25 Correlation Of Vitamin D Levels With Number Of Components

\begin{tabular}{|c|c|c|c|c|c|c|c|c|c|}
\hline & & \multicolumn{6}{|c|}{ VITAMIN D } & \multicolumn{2}{|c|}{ TOTAL } \\
\hline & & \multicolumn{2}{|c|}{$\begin{array}{c}\text { Deficient } \\
(<=20)\end{array}$} & \multicolumn{2}{|c|}{$\begin{array}{c}\text { Insufficient } \\
(21-29)\end{array}$} & \multicolumn{2}{|c|}{$\begin{array}{c}\text { Sufficient } \\
(>=\mathbf{3 0})\end{array}$} & \multirow[t]{2}{*}{ No. } & \multirow[t]{2}{*}{$\%$} \\
\hline & & No. & $\%$ & No. & $\%$ & No. & $\%$ & & \\
\hline \multirow{3}{*}{ No. of comp } & 3.00 & 20 & 57.1 & 10 & 28.6 & 5 & 14.3 & 35 & 100.0 \\
\hline & 4.00 & 43 & 93.5 & 2 & 4.3 & 1 & 2.2 & 46 & 100.0 \\
\hline & 5.00 & 19 & 100.0 & & & & & 19 & 100.0 \\
\hline \multicolumn{2}{|c|}{ TOTAL } & 82 & 82.0 & 12 & 12.0 & 6 & 6.0 & 100 & 100.0 \\
\hline
\end{tabular}

Chi-Square Test

\begin{tabular}{|l|c|c|c|c|}
\hline & Value & df & Sig. & P value \\
\hline Chi-Square & $22.928^{\mathrm{a}}$ & 4 & $* *$ & $\mathrm{P}<0.01$ \\
\hline
\end{tabular}

In patients with 3 components of metabolic syndrome, 20 (57.1\%)patients had deficient vitamin D levels, $10(28.6 \%)$ had insufficient levels and $5(14.3 \%)$ had sufficient levels. In patients with 4 components of metabolic syndrome,43 (93.5\%)patients had deficient vitamin D levels,2(4.3\%) had insufficient levels and $1(2.2 \%)$ had sufficient levels. In patients with all 5 components of metabolic syndrome (19) all had deficient levels. $(\mathrm{p}<0.01)$ 
“A Study Of Vitamin - D Levels In Metabolic Syndrome And Its Association...

Table No : 26 Correlation Of Vitamin D Levels With Stroke

\begin{tabular}{|c|c|c|c|c|}
\hline & & \multicolumn{3}{|c|}{ VITAMIN D } \\
\hline & & Mean & S.D & No. \\
\hline \multirow{2}{*}{ Stroke } & No & 17.01 & 6.28 & 82 \\
\hline & Yes & 10.33 & 2.11 & 18 \\
\hline \multicolumn{2}{|c|}{ Total } & 15.81 & 6.30 & 100 \\
\hline
\end{tabular}

t-test for Equality of Means

\begin{tabular}{|c|c|c|}
\hline $\mathrm{t}$ & $\mathrm{df}$ & Sig. \\
\hline 4.441 & 98 & $<0.01$ \\
\hline
\end{tabular}

$\mathrm{T}$ test shows there is significant decrease in vitamin $\mathrm{D}$ levels in patients who developed stroke when compared to those without stroke. $(\mathrm{p}<0.01)$

Table no : 27correlation of vitamin d levels with myocardial infarction:

\begin{tabular}{|c|c|c|c|c|}
\hline \multicolumn{2}{|c|}{} & \multicolumn{3}{|c|}{ VITAMIN D } \\
\cline { 3 - 5 } \multicolumn{2}{|c|}{} & Mean & S.D & No. \\
\hline \multirow{3}{*}{ MI } & No & 16.98 & 6.44 & 80 \\
\cline { 2 - 5 } & Yes & 11.15 & 2.37 & 20 \\
\hline \multicolumn{2}{|c|}{ Total } & $\mathbf{1 5 . 8 1}$ & $\mathbf{6 . 3 0}$ & $\mathbf{1 0 0}$ \\
\hline
\end{tabular}

t-test for Equality of Means

\begin{tabular}{|c|c|c|}
\hline $\mathrm{T}$ & $\mathrm{df}$ & Sig. \\
\hline 3.964 & 98 & $<0.05$ \\
\hline
\end{tabular}

\subsection{Vitamin $d$ and its functions :}

\section{Discussion}

Vitamin D was first identified and characterized in 1923 by Goldbatt and Soames [1]. There are five forms of vitamin D. (D1-D5). The major form vitamin D2 (ergocalciferol) is obtained from ergosterol. The sources of vitaminD2 are plants and invertebrates which synthesis vitaminD2 in responses to sunlight. They are not synthesized by vertebrates. Vitamin D3 (cholecalciferol) is obtained from dehydrocholesterol. It is synthesized in skin when 7-Dehydrocholesterol reacts with ultraviolet (UV) radiation to form vitamin D3.In humans the major source, about $80-90 \%$ of vitamin D comes from skin on exposure to sunlight and the dietary source of vitamin D is only 10-20\% [2]. Vitamin D performs wide range of biological functions in the body through Vitamin D receptors which are expressed in tissues including skeletal muscles, vascular smooth muscles, osteoblasts, endothelium, cardiomyocytes, activated macrophages and pancreatic beta cells. Vitamin D regulates calcium and phosphorous homeostasis. It enhances calcium and phosphorus absorption in the gut and promotes bone mobilization [3]. In addition to calcium homeostasis, VDR also stimulate insulin production and modulate immune function [4] including inhibiting angiogenesis, inhibiting renin production and stimulating macrophage ecathelicidin production [5].

Serum concentration of 25 -hydroxy vitamin D reflects the vitamin D status and has longer half life than calcitriol. 1, 25-OH vitamin D levels in the circulation may be normal or elevated in vitamin D deficient patients and hence it should not be used to determine vitamin D status .IOM (Institute Of Medicine) and the Endocrine society have defined vitamin D deficiency as 25-hydroxy vitamin D less than $20 \mathrm{ng} / \mathrm{ml}[6,7]$.Vitamin $\mathrm{D}$ insufficiency has been defined as 25-hydroxy vitamin D of $21-29 \mathrm{ng} / \mathrm{ml}$ and concentrations of $>30 \mathrm{ng} / \mathrm{ml}$ is considered to be sufficient. Excess vitamin D concentration is also undesirable [8]. The upper limit of vitamin D is about $80 \mathrm{ng} / \mathrm{ml}$. Serum vitamin D concentration of $>200 \mathrm{ng} / \mathrm{ml}$ is considered potentially toxic [9].Vitamin D can be measured using the following techniques.

1. Immunoassay technique-Radioimmunoassay (RIA) and Chemiluminescence immunoassay

2. Chromatography techniques-High pressure liquid chromatography (HPLC) and liquid chromatography coupled with tandem mass spectrometry (LC-MS).

Vitamin D insufficiency occurs because of inadequate exposure to sunlight. It is common among elderly, hospitalized and institutionalized persons. In addition to this, other factors related to vitamin D deficiency include dark skin colour, increased distance from equator, winter season, clothing, sunscreen, air pollution, smoking, malabsorption, obesity, diseases of renal, liver and some drugs [10]. Vitamin D deficiency in children causes rickets and growth retardation. In adults, it will precipitate osteopenia and osteoporosis. In recent decades, numerous studies have demonstrated the association between vitamin D deficiency with many diseases including diabetes mellitus, systemic hypertension, dyslipidemia, cerebrovascular dieases, cardiovascular disorders, metabolicsyndrome, cancer, infections, chronic kidney disease, tuberculosis and autoimmune disorders [11]. 
The American academy of paediatrics recommends $400 \mathrm{IU}$ as minimum requirement of vitamin $\mathrm{D}$ for all infants and children. The National osteoporosis foundation recommends 400 IU -800 IU daily for adults under the age of 50 years and 800-1000 IU daily for adults older than 50 years. The North American menopause society recommends 700-800 IU daily for women at risk of deficiency due to low sun exposure. Guidelines from the osteoporosis society of Canada recommended vitamin D 400 IU per day for people upto the age of 50 years and $800 \mathrm{IU} /$ day for people over 50 years [12] .

Vitmain $\mathrm{D}$ is sequestrated in subcutaneous fat and hence in obese persons vitamin $\mathrm{D}$ stores are less available to become biologically active. Thus there is decreased bioavailability of vitamin D in obesity. Besides this, obese individuals may have sedentary life style and less outdoor activities and thus impaired skin production of vitamin D [13]. Vitamin D deficiency is associated with stroke and the size of infarct. Neuro protective role of vitamin D also has been reported [14]. Vitamin D is associated with several other diseases like peripheral vascular disease, renal diseases, cancer, autoimmune diseases, tuberculosis and cardiovascular diseases including cardiac failure, myocardial infarction. Recently hypovitaminosis D has been recognized as a potential risk factor for cardiovascular events like myocardial infarction, stroke and congestive cardiac failure.. Low serum vitamin D levels are linked with diminished coronary flow, endothelial dysfunction, higher coronary artery calcium scores and increased vascular stiffness and increased mean platelet volume [15].Vitamin D has cardioprotective role in post myocardial infraction patients via antiapoptotic, anti-inflammatory and antifibrotic mechanisms [16]. Serum vitamin D levels are inversely correlated with TGL (Triglycreides) and VLDL (Very low density lipoprotein) levels. Lipid profile abnormality in vitamin D deficiency occurs because of peripheral insulin resistance. Vitamin D deficiency also increases oxidation of LDL and free radicals. Vitamin D has suppressive effect on serum parathyroid hormone (PTH) concentration. Low PTH causes increased peripheral clearance of TGLs and thus reducing serum TGL levels [17].

The role of vitamin D in Diabetes is initially suggested by seasonal variation in glycemic control in diabetic patients with worse in winter, that may be due to vitamin D deficiency as a result of reduced sunlight in winter.Vitamin D deficiency inhibits the insulin secretion from pancreas and its turnover which leads to impaired glucose tolerance [18] .Insulin resistance is regarded as precursor in development of Diabetes Mellitus. Vitamin D stimulates the expression of insulin receptors and hence enhances insulin mediated glucose transport. It also enhance insulin action indirectly by regulating the extracellular calcium levels [19]. Vitamin D suppresses the expression of renin. Vitamin D also have direct effects on vascular structure by its antiproliferative property on smooth muscle cells of vessel. A study by Forman et.al reported an increase in the relative risk of hypertension in vitamin D insufficient subjects [20].

\subsection{Metabolic syndrome :}

Metabolic syndrome previously known as syndrome X or insulin resistant Syndrome is a constellations of factors that link obesity, hypertension, Insulin resistance (IR), Type II DM and cardio vascular disease [11].

IDF (international diabetes foundation) criteria for diagnosis of metabolic syndrome [21]

Central obesity defined as waist circumference :

\begin{tabular}{|c|l|l|}
\hline MEN & \multicolumn{1}{|c|}{ WOMEN } & \multicolumn{1}{c|}{ ETHNICITY } \\
\hline$>94 \mathrm{~cm}$ & \multicolumn{1}{|c|}{$>80 \mathrm{~cm}$} & $\begin{array}{l}\text { Europoid,subsaharahafrican,easternand middle } \\
\text { eastern. }\end{array}$ \\
\hline$>90 \mathrm{~cm}$ & $>80 \mathrm{~cm}$ & $\begin{array}{l}\text { South Asian,Chinese, and ethnic south and } \\
\text { central American. }\end{array}$ \\
\hline$>85 \mathrm{~cm}$ & $>90 \mathrm{~cm}$ & Japanese \\
\hline
\end{tabular}

\section{Plus atleast two of the following:}

$>\quad$ Fasting triglycerides $>150 \mathrm{mg} / \mathrm{dl}$ or specific medication.

$>$ HDL cholesterol $<40 \mathrm{mg} / \mathrm{dl}$ in men and $<50 \mathrm{mg} / \mathrm{dl}$ in women or on specific medication.

$>$ Blood pressure $>130 \mathrm{~mm}$ of $\mathrm{Hg}$ systole or $>85 \mathrm{~mm}$ of $\mathrm{Hg}$ diastolic or previous diagnosis or specific medication

$>\quad$ Fasting plasma glucose $>100 \mathrm{mg} / \mathrm{dl}$ or previously diagnosed type 2 diabetes mellitus.

Of the numerous metabolic syndrome definitions, the NCEP-ATP III is commonly used in research. According to this definition, the presence of atleast 3 of the following five confirms having metabolic syndrome for adults.

NCEP: ATP (National Cholesterol Education Programme : Adult Treatment Panel ) III 2001 criteria for metabolic syndrome: [22]

- Central obesity - defined as waist circumference of $>102 \mathrm{~cm}$ in males $>88 \mathrm{~cm}$ in females.

- Hypertriglyceridemia - defined as serum triglyceride levels of $>150 \mathrm{mg}>\mathrm{dl}$ or on specific medication. 
- Low HDL cholesterol defined as $<40 \mathrm{mg} / \mathrm{dl}$ in males and $<50 \mathrm{mg} / \mathrm{dl}$ in females or specific medication

- Hypertension defined asbBlood pressure $>130 \mathrm{~mm}$ of $\mathrm{Hg}$ systolic or $>85 \mathrm{~mm}$ of $\mathrm{Hg}$ diastolic or on specific medication

- Fasting blood glucose $>100 \mathrm{mg} / \mathrm{dl}$ or on specific medication or previously diagnosed type 2 diabetes mellitus.

\subsubsection{Pathophysiology of metabolic syndrome:}

Metabolic syndrome is characterized by several abnormalities including impaired glucose tolerance, blood lipid derangements and prothrombotic and pro-inflammatory states. Adipose tissue dysfunction plays major role in pathogenesis of obesity and insulin resistance. Enlargement of adipose tissue and macrophage infiltration of the adipose tissue leads to cytokine release and favours insulin resistance. Insulin resistance seems to be the major mediator of metabolic syndrome.A number of studies suggested obesity visceral obesity as the culprit in metabolic syndrome. The visceral fat secretes inflammatory adipokines and all components of Renin angiotensin system(RAS). The RAS regulates BP and excessive RAS stimulation lead to hypertension. The RAS is upregulated in obesity $[23,24,25]$. The adipocytokines secreted by adipose tissue are leptin, resistin, IL6, plasmogen activator inhibitor-1, TNF alpha. Increased concentrations of TNF alpha and IL-6 stimulates production of acute phase protein, $\mathrm{C}$ reactive Protein(CRP) in liver. High concentration of CRP indicate systemic inflammation and contribute to atherosclerotic plaque development [26]. Hence increase in adiposity triggers cascade of events including RAS activation and increased secretion of pro-inflammatory adipcytokine leading to insulin resistance, atherogenic dyslipidemia. Other factors like oxidative stress, hereditary and life style factors also contribute development to metabolic syndrome.The risk factors include overweight/obesity, sedentary lifestyle, ageing, diabetic mellitus and lipodystrophy. The family history also a role in pathogenesis of metabolic syndrome. No particular gene has been implicated yet, which suggest that environment place substantial influence [27].

\subsubsection{Others disorders associated with metabolic syndrome:}

Non alcoholic fatty liver disease (NAFLD), hyperuricemia, poly cystic ovarian Syndrome (PCOS), obstructive sleep apnoea syndrome are some other conditions associated with metabolic syndrome [22].

\subsubsection{Complications of metabolic syndrome :}

Cardiovascular complications are mainly associated with metabolic syndrome. They include coronary artery disease, atrial fibrillation, heart failure, ischemic stroke, veno thromboembolic disease. NAFLD is another important condition associated with metabolic syndrome. Breast carcinoma is also linked to metabolic syndrome because of plasminogen activator inhibitor-1 cycle dysregulation. Studies have suggested that cancers of colon, kidney, gallbladder, prostate gland are also linked with metabolic syndrome recent studies shows association of metabolic syndrome and psoriasis. When there is increase in number of components of metabolic syndrome, the risk of recurrent preeclampsia is also increased. Accelerated cognitive aging is also associated with metabolic syndrome [28].

\subsubsection{Treatment:}

Lifestyle modifications and weight reduction are the initial steps in the management of metabolic syndrome.Diets rich in fruits, vegetables, cereal grains and fish should be encouraged.Diet enriched in saturated fats, transfatty acids and cholesterol should be restricted. Regular moderate intensity physical activity for atleast 30 minutes for atleast 5 days per week should be followed. Inspite of lifestyle intervention, weight loss drugs may be needed in some patients. They can be absorption inhibitors and appetite suppressors. Appetite suppressants include sibutramine and phentermine. Orlistat inhibits absorption of fat in about $30 \%$. Bariatric surgery may be a option in metabolic syndrome patients with BMI $>40 \mathrm{~kg} / \mathrm{m}^{2}$ and $>35 \mathrm{~kg} / \mathrm{m}^{2}$ with other comobidities. Statins are used to lower LDL cholesterol Ezetimibe can be used as a second option which reduces 15-20\% LDL cholesterol. Cholestyramine and colestipol are the bileacids sequestrants that can be tried but they often increase triglyceride levels and hence, should be used with caution in metabolic syndrome patients. Nicotinic acid also lowers LDL cholesterol by less than $20 \%$. Fibrates like fenofibrate and gemfibrozil can be tried when both triglycerides and LDL cholesterol are elevated. Nicotinic acid is the only available drug with predictable HDL cholesterol raising properties. ACE inhibitors or Angiotensin II receptor blockers are recommended for patients with metabolic syndrome and without diabetes mellitus as they reduce the incidence of new onset diabetes mellitus. Aggressive glycemic control is essential as it helps in modification of triglyceride levels and HDL cholesterol levels. Metformin can be used for hyperglycemia in metabolic syndrome patients and some literature says that it helps to reverse certain pathologic changes in metabolic syndrome. Aspirin can be given for primary prevention of cardiovascular complications [29] . 


\subsection{Vitamin $D$ and metabolic syndrome:}

Vitamin D influences several components of metabolic syndrome like hypertension, hyperglycemia, insulin resistance and hyperlipidemia. The immunomodulatory effect of vitamin D and its inverse association with inflammation suggest that role of vitamin D in metabolic syndrome. Metabolic syndrome is usually associated with low grade systemic inflammation and oxidative stress. These two factors are belived to have important role in development of metabolic syndrome itself and its future adverse effects. Vitamin D by its antiinflammatory and oxidative stress attenuating effect suggested to play a vital role in pathogenesis of metabolic syndrome. Insulin reistance being a major underlying mechanism in metabolic syndrome, most epidemiological studies have demonstrated that serum $25(\mathrm{OH}) \mathrm{D}$ and VDR polymorphism are associated with insulin sensitivity. NHANES study 2003-2004 studies have demonstrated that serum vitamin D levels are significantly negatively correlated with metabolic syndrome and also with each of its individual components. Some studies suggested that maintaining optimal level of vitamin D may provide protective effects against metabolic syndrome and its sequel. In a study done by Ling Lu, et al, middle aged and elderly Chinese individuals demonstrated that low vitamin D levels are significantly associated with increased risk of having metabolic syndrome and adverse values for individual metabolic components. However no data is currently available on effects of vitamin D supplementation and prevalence and treatment of metabolic syndrome in vitamin D deficient patients.Vitamin D levels also predict all cause and cardiovascular mortality in patients with metabolic syndrome. It was evidenced by prospective cohort study done in 1801 metabolic patients who underwent elective coronary angiography between 1997 and 2000. Mortality was tracked for a median of 7.7 years. The results was that there was 75\% reduction in the hazard ratio in the patients with optimal vitamin D levels compared to those with severe deficiency. This study also demonstrated serum level dependent reduction in cardiovascular mortality $[30,31,32,33]$.

\subsection{Current study and previous studies :}

In our study there is significant decrease in vitamin $\mathrm{D}$ levels with increasing age similar to the study done by Dalgard C et al[34], where they observed significant low levels of vitamin D with increasing age. With regard to gender, there is no significant difference in vitamin D levels similar to the observation by Ford ES et al [35] . There is significant decrease in vitamin $\mathrm{D}$ levels with increase in duration of diabetes. This is contrast to the study done by Braun TR et al [36] where they observed no relation between duration of diabetes and vitamin D levels.

Vvitamin D levels were not significantly associated with fasting blood glucose levels in our study. Many cross sectional studies with subjects of varying age groups in non diabetic and diabetic states metabolic syndrome have demonstrated significant association between insulin resistance and low serum vitamin D levels. In a study done by Afsaneh Talari et al [37] where they noted significant reduction in fasting plasma glucose after increased intake of vitamin D. In this study 100 diabetic patients received 50000units of vitamin D3 orally per week for about 8 weeks. They also shadowed significant improvement in serum insulin, HOMA- IR after treatment. Thus suggesting that vitamin D supplementation may reduce IR in diabetes. In a cross sectional study done by Hala Ahmadieh et al [38] 136 diabetic patients participated. They noted that vitamin D is negatively correlated with BMI and age but not with duration of diabetes. They showed that low vitamin D levels were highly prevalent in diabetic patients, similar to those observed in our study here.

In our study, waist circumference was negatively correlated with vitamin D levels. We observed significant association between hypertension and vitamin D levels. It is similar to the study done by Chon SJ et al [39] where blood pressure is negatively correlated with vitamin D levels. In our study among the lipid profiles, triglycerides and total cholesterol were significantly inversely correlated with serum levels of vitamin D. This is similar to observed by the study done by Kedam, Durgaprasr et al [33] where they showed significant inverse relation of vitamin $\mathrm{D}$ with triglycerides.

In our study 38 patients developed cardiovascular complications of metabolic syndrome in the form of stroke and myocardial infraction. Stroke occurred in 18 patients and myocardial infarction occurred in 20 patients. They are significantly associated with low vitamin D levels. This is similar to those observed in the cohort study done by Wang and colleagues [40]. They studied 1739 Framingham offspring participants without any prior history of cardiovascular disease. They finally concluded that low vitamin D levels were significantly associated with increased incidence of cardiovascular events during the follow up for mean of 5.4 years.

\section{Conclusion}

The present study was conducted to estimate serum vitamin D levels among metabolic syndrome patients admitted in Coimbatore medical college hospital and to study the possible association of vitamin D levels with various factors. The study included 100 metabolic syndrome patients with no factors significantly altering the vitamin D levels. Vitamin D levels were significantly low among metabolic syndrome patients and there was significant association with duration of diabetes, hypertension, abnormal waist circumference, 
triglyceride and total cholesterol levels. However we did not find significant association between vitamin D levels and other factors like fasting blood sugar, HDL and LDL cholesterol levels. At the same time, vitamin D levels were significantly low in patients with complications of metabolic syndrome like myocardial infarction and stroke compared to those without complications. Thus it may be useful in clinical practice to measure vitamin D levels in patients with metabolic syndrome and it can be used to predict complications of metabolic syndrome like myocardial infarction and stroke. Further studies are needed to assess the preventive role of Vitamin D in metabolic syndrome and its complications.

\section{References}

[1]. DeLuca HF. Overview of general physiological features and functions of vitamin D. Am J ClinNutr 2004;80(6 suppl) : 1689s-96s.

[2]. Webb AR, Kline L Holick MF (1998) Influence of season and latitude on the cutaneous synthesis of vitamin D 3 : Exposure to winter sunlight in Boston and Edmonton will not promote vitamin D3 synthesis in human skin . J Clin Endocrinolmetab 67:373378.

[3]. Holick MF. Sunlight and vitamin D for bone health and prevention of autoimmune disease, cancer and cardiovascular disease. Am J Clin Nutr. Dec 2004:80 (6 suppl):16785-16888.

[4]. Tsoukas CD, Provvendini DM, Manoglas SC.1,25 dihydroxy vitamin D3 : a novel immune regulatory hormone. Science Jun 29 1984;224(4656):1438-1440.

[5]. Adams JS, Hewison M 2010 Update in vitamin D . J Clin Endocrinolmetab 95:471-478

[6]. Holick MF 2007 Vitamin D deficiency. N Engl J med 357:266-281.

[7]. Malabanan A, Veronikis IE, Holick MF 1998 redefining vitamin D insufficiency. Lancet 351:805-806.

[8]. Misra M ,Pacaud D ,PetrykA, Collett-solberg PF, KappyM. Vitamin D deficiency in children and its management: review of current knowledge and recommendations. Paediatrics. Aug 2008;122(2):398-417.

[9]. Wang TJ, PencinaMJ, BoothSL,et.al. Vitamin D deficiency and cardiovascular disease. Circulation, Jan 29 2008;117(4):503-511.

[10]. Harris SS, soteriades E, Coolidge JA, mudgal S, Dauson - Hughes B. Vitamin D insufficiency and hyperparathyroidism in a low income, multiracial, elderly population. J Clin Endocrinol Metab2000; $85: 4125-4130$.

[11]. Defronzo RA ,Ferrannini E .Insulin resistance, a multi faceted syndrome responsible for NIDDM, obesity, hypertension, dyslipidemia and atherosclerotic cardiovascular disease. Diabetescare. Mar 1991;14(3):173-194.

[12]. IOM 2011 Dietary reference intakes for calcium and vitamin D .Washington DC: The National Academic Press.

[13]. J. Wortsman, L. Y. Matsuoka, T. C. Chen, Z. Lu, and M. F. Holick, "Decreased bioavailability of vitamin D in obesity," The American Journal of Clinical Nutrition, vol. 72, no. 3, pp. 690-693, 2000.

[14]. S. Pilz, A. Tomaschitz, C. Drechsler, A. Zittermann, J. M. Dekker, and W. März, "Vitamin d supplementation: a promising approach for the prevention and treatment of strokes," Current Drug Targets, vol. 12, no. 1, pp. 88-96, 2011. View at Publisher - View at Googl

[15]. V. Kunadian, G. A. Ford, B. Bawamia, W. Qiu, and J. E. Manson, "Vitamin D deficiency and coronary artery disease: A review of the evidence," American Heart Journal, vol. 167, no. 3, pp. 283-291, 2014.

[16]. S. Bae, S. S. Singh, H. Yu, J. Y. Lee, B. R. Cho, and P. M. Kang, "Vitamin D signaling pathway plays an important role in the development of heart failure after myocardial infarction," Journal of Applied Physiology, vol. 114, no. 8, pp. 979-987, 2013.

[17]. Lind L et al. Vitamin D is related to blood pressure and other cardiovascular risk factors in middle aged men. Am J Hypertens 1997 $137: 495-502$

[18]. Norman AW, Frankel JB, Heldt AM, Grodsky GM Vitamin and deficiency inhibits pancreatic secretion of Insulin. Science. 1980 ; 209 (4458); 823-5.

[19]. Seshadrie Ka, Tamil Selvana B, Rajendrana A. Role of Vitamin D in Diabetes. J EndocrinolMetab 2011; 1(2) ; 47-56.

[20]. Forman JP, Giovannucci E, Holmes MD, et al. Plasma 25 - hydroxyl vitamin D levels and risk of incident hypertension. Hypertension $2007 ; 49: 1063-1069$.

[21]. Alberti K G. Zimmet P, Sha J .Metabolic syndrome - a new world wide definition A consensual statement for the international diabetes federation. Diabet Med 2006;23(5):469-80.

[22]. Executive Summary of The Third Report of The National Cholesterol EducationProgram (NCEP) Expert Panel on Detection, Evaluation, And Treatment of HighBlood Cholesterol In Adults (Adult Treatment Panel III). JAMA. May 16 2001;285(19):24862497.

[23]. Third report of the NCE expert panel on detection ,evaluation and treatment of high blood cholesterol in adults (Adult Treatment Panel III)final report. Circulation.2002;106(25):3143-3421.

[24]. Morse SA, Zhang R, Thakur V, Reisin E. Hypertension and the metabolic syndrome. Am J Med Sci Dec 2005; 330(6) ; 303-310.

[25]. Despres JP, Lemieux I, Bergeron J, et al. Abdominal obesity and the metabolic syndrome : contribution to global cardio metabolic risk. Arterioscler Thrombvasc Biol. 2008 : 28(6) ; 1039-1049.

[26]. De Ferranti SD, Rifai N. C-reactive protein : a non traditional serum marker of cardiovascular risk. Cardiovascpathol. Jan feb2007 ; 16(1) " 14-21

[27]. Potenza MV, Mechanick JI. The metabolic syndrome ; definition, global impact, and pathophysiology. Nutrclin Pract. Oct - Nov $2009 ; 24(5): 560-577$.

[28]. Grundy SM. A constellation of complications: the metabolic syndrome. Clin Cornerstone.2005;7(2-3):36-45

[29]. Grundy SM et al. Diagnosis and Management of the Metabolic Syndrome. An American Heart Association/National Heart, Lung and Blood Institute Scientific Statement. Circulation 2005;112:2735-2752.

[30]. Lu, Ling, Zhijie Yu, An Pan, Frank B. Hu, Oscar H. Franco, Huaixing Li, Xiaoying Li, Xilin Yang, Yan Chen, and Xu Lin. 2009. Plasma 25-hydroxyvitamin D concentration and metabolic syndrome among middle-aged and elderly Chinese individuals. Diabetes Care 32(7): 1278-1283.

[31]. Vitezova A et al vitamin D status and metabolic syndrome in the elderly: Rotterdam study .Eu J Endocrinol 2015 Mar;172(3):32735.

[32]. Inka Minambres et al The Association of hypovitaminosis D with the metabolic syndrome is independent of the degree of obesity. ISRN Endocrino,.2012:691803.

[33]. Kedam Durga Prasad P, Havilah, Pandit Vinodh. A study of vitamin D and metabolic syndrome in urban population. Int J Biol Med Res.2012;3(2):1731-1734.

[34]. Dalgard C ,Petersen MS, WeiheP, Grandjean P. vitamin D status in relation to glucose metabolism and type 2 diabetes in septuagenerians. Diabetes care 2011;34(6):1284-8. 
[35]. Ford ES, Ajani U A, Mc Guire LC, Liu S .concentrations of serum vitamin D and metabolic syndrome among US adults. Diabetes care 2005;28(5):1228-30.

[36]. Braun TR, Been L F, Blackett P R, Sanghare D K. Vitamin D deficiency and cardio metabolic risk in north Indian community with high prevalent type 2 diabetes. J Diabetes Metab 2013;3:100213.

[37]. Afsanesh Tet al The effect of vitamin D on insulin resistance in patients with type 2 diabetes. Diabetology \& Metabolic Syndrome 2013, 5:8

[38]. Hala Ahmadieh, Sami T. Azar, Najla Lakkis, and Asma Arabi Hypovitaminosis D in Patients with Type 2 Diabetes Mellitus : A Relation to Disease Control and Complications, ISRN Endocrinology.

[39]. Chon SJ, Yun BH, Cho SH et al. (2014) Association between Vitamin D Status and Risk of Metabolic Syndrome among Korean Postmenopausal Women. PLoS ONE 9(2): e89721. doi:10.1371.

[40]. Wang TJ ,Pencina M J, Booth S L, et al. Vitamin D deficiency and risk of cardiovascular disease. Circulation.2008;117:503-511 